- General dental practitioner research networks are capable of producing and analysing large and complex datasets.

- Patients' subjectively perceived oral health is affected by the attributes and attitudes of their dentist.

- Longitudinal studies of patients receiving treatment from GDPs would provide important data about the effectiveness of dental care.

\title{
A general dental practice research network: impact of oral health in general dental practice patients
}

\author{
E. J. Kay, ${ }^{1}$ N. Ward ${ }^{2}$ and D. Locker ${ }^{3}$
}

\begin{abstract}
Objective To measure the subjective impact of oral health in a group of patients attending general dental practices in the North West of England and to investigate the attributes of dentists and practices in order to examine how such attributes might relate to patients' subjective perceptions of oral health.

Design Fifteen general dental practices conducting a simultaneous survey of attending patients and 15 practitioners from these practices providing information about their attitudes to treatment, prevention and various aspects of their surgery.

Setting General dental practice

Outcome measures Patient subjective impact scores. Relationships between practice and practitioner variables and patients' subjectively perceived oral health.

Results Fifteen practitioners with diverse practice attributes provided data on 718 patients. The mean total oral health impact score was 18.4. Twenty two per cent of patients had experienced pain in the four weeks before the survey and $11 \%$ had been unable to chew some foods. Fifty five per cent of the surveyed population had, in the previous year, worried about the appearance of their mouth and 65\% had worried about their oral health in general. Dentists' beliefs were related to patient impact scores but practice attributes were not significantly associated with patients' impacts.

Conclusions Fourteen percent of the differences in patients' subjectively perceived oral health can be attributed to dentist attitudes and attributes. Further research regarding the influence of dentists personality and professional beliefs on patients well-being needs to be undertaken.
\end{abstract}

Research networks in healthcare play a number of important roles. Firstly, by equipping practitioners with skills, along with the confi-

\footnotetext{
1*Professor of Dental Health Services Research, University of Manchester Dental Hospital and School, Higher Cambridge Street, Manchester M15 6FH; ${ }^{2}$ Associate Postgraduate Dental Dean, Department of Postgraduate Medicine and Dentistry, Gateway House, Piccadilly South, Manchester, M60 7LP; ${ }^{3}$ Director, Community Dental Research Unit, University of Toronto, Toronto, Canada

To contact the North West Dental Practice Research Network this is c/o Gateway House

Gateway House, Piccadilly South, Manchester M60 7LP

${ }^{*}$ Correspondence to: E. Kay

E-mail:e.kay@man.ac.uk
}

\section{Refereed paper}

Received 01.10.02; Accepted 31.01.03

๑ British Dental Journal 2003; 194: 621-625 dence and support required to undertake studies, research networks can improve the quality of primary care research. There is also a shortage of research capacity among primary care practitioners. This is partly because most are simply too busy caring for patients to have the necessary time and resources to undertake research. Furthermore, many of the individuals who enjoy and succeed in research are drawn towards academia. The second role of primary care research networks is therefore to provide a mechanism whereby research capacity and capability within the primary care setting are increased. The third role potentially played by primary care research networks is perhaps more subtle although possibly easier to achieve. Reflective practice, continual self-audit and performance monitoring are key components of improved clinical decision making ${ }^{1}$ and enhanced treatment planning. So, by encouraging practitioners to measure and monitor outcomes of relevance to patients, research networks can help to develop the culture of enquiring practice.

A previous paper ${ }^{2}$ described the philosophy and activities of a dental practice research network. The network was considered highly successful by the involved practitioners. However, it is generally accepted in management science ${ }^{3}$ that the 'success' of a 'network' of any description should be evaluated, not only by the extent to which informal trust-based relationships are formed among the participants but also by the extent to which the group act together to produce relevant research.

Through involvement in a series of research workshops, the network practitioners piloted a subjective oral health status questionnaire in their practices. The network then developed and designed a number of research plans, using the piloted questionnaire. The practitioners selected and developed a research plan and the group developed this into a full research protocol. The research and analysis, carried out by the network, is the subject of this paper.

Instead of focusing on the amount of disease patients had, the practitioners wanted to investigate the extent to which their patients' oral health caused their patients worry, concern, social limitation or symptoms. The development of patient-based, as opposed to dentist-based, indicators of health is part of a broad paradigm shift, in which the psychosocial model of health has replaced the medical model. ${ }^{4}$ Several indicators of oral health have been posited, ${ }^{5}$ but in general all reflect, or describe in some way, the functional, social and psychological outcomes of oral disorders 
and conditions. ${ }^{6}$ Most subjective indicators of oral health status are based on a model of disease and its consequences derived from the World Health Organisation's International Classification of Impairments, Disabilities and Handicaps ${ }^{7}$ and the final questionnaire used in this study reflected the key components of that model.

\section{AIM OF THE NETWORK RESEARCH}

The aim of the practitioners research project was to:

1. Measure the subjective impacts of oral health on patients.

2. Investigate attributes of dentists and practices, which might affect patient care and well-being.

\section{METHOD}

Oral health impact questionnaires which had been the subject of one of six research workshops held by the network were delivered by a research fellow in batches of 50 to each of the network practices. The receptionist was asked to stamp each questionnaire with a practice identification stamp and a patient identification code. Each practice was asked to complete a form, identifying patients by both identification code and by name. This obviated any medico-legal or ethical problems concerning patient confidentiality. However, longitudinal studies might re-identify participating patients at a later date if required, but anonymity for the patients and dentist was ensured when the data were analysed.

The practices were asked only to distribute questionnaires to patients over 18-years-old. They were also asked to record on the form if patients did not wish to complete the questionnaire. The practices were asked to collect data on a specific week. They were instructed to issue a questionnaire to each consecutively-attending patient, regardless of the reason for attendance.

A practice questionnaire was completed 6 months subsequent to the survey to measure the dentists' restorative thresholds, attitudes to treatment and prevention, participation in postgraduate education and employment of professions complementary to dentistry in the practice. This was sent by post to dentists six months subsequent to the final workshop with a reply paid envelope. The dentists were reimbursed $£ 50$ for the time spent completing this rather detailed questionnaire.

Statistical data was undertaken using STATA (StataCorp 1999. Stata Statistical Software: Release C.Q College Station TX: Stata Corporation) and SPSS (SPSS Inc. SPSS for Windows Release 10.0, 1999) statistical packages.

Descriptive statistics, $\chi^{2}$, Spearman Rank Correlation and Mann Whitney $U$ tests were used where appropriate. Stepwise linear regression models were fitted to examine the contribution of the dentists' variables to patient oral well-being.

\section{RESULTS}

\section{Dentist participants}

One dentist from each of 15 dental practices participated in the research workshops and the multicentre research programme. Ten were men and five women. Graduation year ranged from 1966 to 1991 (median 1983). They had been practising for between 10 and 34 years (median 19). All reported that they have participated in continuing education courses (min. 7.5 hours, max. 250 hours, median 30 hours) in the previous twelve months. All participants treated more than 50 patients each week whilst $9(60 \%)$ saw over 100.

\section{Dentists' beliefs}

Ten of the dentists believed that caries in primary teeth progressed from outer enamel to ADJ in less than 12 months, whilst 12 of the 15 dentists believed that caries in permanent teeth took longer than 1 year to reach the $\mathrm{ADJ}$.
Table 1 Dentists' opinions regarding recall intervals for patients in different age groups

\begin{tabular}{|c|c|c|c|c|c|c|}
\hline & & & Interval in mont & & & \\
\hline & Age & 4 & 6 & 12 & 18 & $\begin{array}{l}\text { no } \\
\text { specific } \\
\text { interval }\end{array}$ \\
\hline (a) & $3-5$ years & $3(20 \%)$ & $10(66.7 \%)$ & $2(13.3 \%)$ & & \\
\hline (b) & $6-12$ years & $5(33.3 \%)$ & $9(60 \%)$ & $1(6.7 \%)$ & & \\
\hline (c) & 13-18 years & $2(13.3 \%)$ & $11(64.7 \%)$ & & $1(5.9 \%)$ & $2(13.3 \%)$ \\
\hline (d) & $19-30$ years & & $10(66.7 \%)$ & $2(13.3 \%)$ & $1(6.7 \%)$ & $2(13.3 \%)$ \\
\hline (e) & $31-44$ years & & $8(53.3 \%)$ & $5(33.3 \%)$ & $1(6.7 \%)$ & $1(6.7 \%)$ \\
\hline (f) & 45-64 years & & $8(53.3 \%)$ & $6(40.0 \%)$ & $1(6.7 \%)$ & \\
\hline (g) & 65 years & $1(6.7 \%)$ & $7(46.7 \%)$ & $5(33.3 \%)$ & $2(13.3 \%)$ & \\
\hline
\end{tabular}

Table 2 Dentists views as to the importance of various factors on decision making where: 1 = Very important, $5=$ Not at all important

\begin{tabular}{lll}
\hline Factor & & Mean (SD) \\
\hline $\mathrm{A}$ & Your own professional values and preferences & $1.36(0.84)$ \\
\hline $\mathrm{C}$ & The patient's type and amount of past treatment & $1.87(0.92)$ \\
\hline $\mathrm{D}$ & The presence or absence of dental insurance & $4.47(0.92)$ \\
\hline $\mathrm{E}$ & Yourient's ability to pay & $3.00(0.85)$ \\
\hline $\mathrm{F}$ & The patient's oral hygiene practices and oral cleanliness & $1.73(0.70)$ \\
\hline $\mathrm{G}$ & $\begin{array}{l}\text { The proven effectiveness by clinical studies of one procedure } \\
\text { over another }\end{array}$ & $1.53(0.52)$ \\
\hline $\mathrm{H}$ & Your clinical experience & $1.27(0.46)$ \\
\hline $\mathrm{J}$ & The regularity of the patient's attendance pattern & $2.07(0.70)$ \\
\hline $\mathrm{J}$ & $\begin{array}{l}\text { The patient's convenience if the therapy requires frequent } \\
\text { recalls and time }\end{array}$ & $2.20(0.77)$ \\
\hline $\mathrm{K}$ & The patient's financial circumstances & $2.67(1.05)$ \\
\hline $\mathrm{L}$ & Whether the patient is new or a regular patient & $2.87(1.36)$ \\
&
\end{tabular}

When asked how long one of their own mesio-occlusal amalgams in adult patient could be expected to last, the responses varied between 4 to 10 years (median 9 years). The majority of dentists believed that 6 months was the optimal interval between dental examinations for all age groups apart from patients aged over 65 years (Table 1 ).

The dentists exhibited a belief in preventive methods, with all agreeing that early enamel caries could be cured and all but one dentist agreeing that dental personnel could successfully influence effective brushing with fluoride toothpaste. Eleven of the 15 felt that diet counselling was an ineffective method for preventing caries.

When asked which factors served to influence the dentists' choice of materials and procedures, postgraduate and continuing education courses were rated as the most important source, followed by established guidelines and reviews. Articles in books, mailed advertising and adverts on the World Wide Web were the least important sources of information.

The practitioners stated that their clinical experience and their own values and preferences had the most important influence on their treatment decision making (Table 2). The least important influences reported by the 15 dentists were the patient's ability to pay or the patient's possession of insurance.

\section{Survey of the dentists' patients}

Each of the 15 practices arranged the completion of 50 patient questionnaires. Of these 750 questionnaires, 718 were returned. This represents a 95.7\% return rate. The number returned by each practice ranged from 10 to 50 . The reasons given for non return of questionnaires were: insufficient staff (receptionists and nurses), lack of time prior to the patient being seen and sickness or vacations for the dentist. The questionnaire was similar to, and based on the subjective oral health status index developed by Locker. ${ }^{4}$ 


\begin{tabular}{|c|c|c|c|}
\hline Sub-scale & Mean (SD) & Minimum & Maximum \\
\hline Chewing & $0.33(0.92)$ & 0 & 6 \\
\hline Speaking & $0.21(0.64)$ & 0 & 3 \\
\hline Pain & $1.04(1.37)$ & 0 & 7 \\
\hline Symptoms & $1.42(1.56)$ & 0 & 9 \\
\hline Eating & $4.27(1.97)$ & 3 & 15 \\
\hline Social & $0.53(0.79)$ & 0 & 4 \\
\hline Living & $6.69(2.05)$ & 6 & 22 \\
\hline Worry & 3.88 (1.94) & 2 & 10 \\
\hline Total & $18.37(7.35)$ & 11 & 66 \\
\hline
\end{tabular}

\section{Patient impacts}

The distribution of the subjectively perceived oral health impacts are shown in Table 3.

A higher score indicated more oral health impact. The overall mean total impact score for the 718 patients was 18.37 .

The 718 questionnaire responses showed that $82(11.4 \%)$ of respondents had experienced some impact on their chewing ability with $18(2.5 \%)$ being unable to chew even boiled vegetables. Sixty-one people $(8.4 \%)$ had had speech problems while 235 (32.5\%) had experienced pain with hot and cold foods and 163 $(22.6 \%)$ having experienced toothache within the 4 weeks prior to the study. One hundred and ninety seven (27.3) patients reported having bleeding gums and 140 (19.4) as having ulcers or sores in their mouth in the 4 weeks prior to completing the questionnaire.

One third (237) of the participants stated that their dental health had, in the last 12 months, prevented them from eating foods they would have liked to eat, and approximately one third $(225 ; 31 \%)$ expressed dissatisfaction with the appearance of their teeth/dentures.

Seventy (10\%) of patients had avoided leisure activities including socialising in the year prior to the survey due to pain, discomfort or other problems with their teeth, mouth or dentures, and more than half $(390 ; 55 \%)$ stated that they had at least sometimes worried about the appearance of their teeth and mouth in the previous year. Four hundred and sixty nine (65\%) had worried about their oral health in the 12 months prior to the survey.

Statistically significant differences between age groups were shown by a Kruskall Wallis test. Table 4 shows that chewing impacts were more likely to be experienced by the older age groups but that these older groups were less likely to report pain $(P<$ 0.05). Chewing and speech impacts were shown to be more likely to be experienced by denture wearers.

\section{Relationship between dentists' treatment attitudes and patients' impact scores}

There was a statistically significant negative correlation between dentists' expectations of longevity of an amalgam and their patients' total impact score (Spearman's correlation coefficient $0.575 ; P<0.05)$ and the more positive the dentists' attitude to prevention, the lower the patients' total impact scores. The dentists' feelings about the importance of regularity of attendance on treat- ment decision making were also correlated with total impact score The more influence patients' attendance had on treatment decision making, the lower the impact score, ie dentists whose decisions were not influenced by the patients' attendance history tended to have patients with higher impacts (Sp Corr. 0.816; $P<0.001$ ). A further influence on treatment decision making, which appeared to be associated with higher impact scores was the dentists view of the patients ability to pay (Sp Corr. 0.594; $P<0.05$ ). This shows that the less importance the dentist placed on the patients' ability to pay, the lower that dentist's patients' total impact score.

There was no correlation between the dentists attitudes to under and over treatment and their patients' impact scores. Although no practice characteristics were significantly correlated with patient impact scores, the number of hours worked by hygienists approached significance in a negative correlation, ie lower impact scores tend to be associated with hygienist deployment in a practice.

A regression model analysed the independent effects of the variables revealed in the univariate analysis as having a significant effect (Table 5). The overall $\mathrm{R}^{2}$ for the model was 0.14 . However, three factors were found to have a significant effect when other variables were controlled for. These were: the extent to which the dentist altered his treatment in relation to the patients' regularity of attendance, the number of years practising and the number of hours spent attending postgraduate education.

Table 5 shows that dentists who placed less importance on their patients' attendance patterns when planning treatment had patients with high mean impact scores It also shows a weak but significant correlation illustrating that dentists who had been in practice longer tended to have patients with higher overall mean impact scores. The fifth row in Table 5 demonstrates a weak negative association between the number of hours spent in postgraduate education and mean patient impact.

\section{DISCUSSION}

The demographic profile of the dentists involved in this study indicated that a wide variety of practitioners took part. There were no very recently qualified dentists but the proportion of men and women reflected the gender distribution within general dental practice as a whole. The sample was not intended to be representative. Rather, it was hoped that the voluntary nature of participation would offer access to diverse practice populations. The fact that there were substantial differences in the 'types' of practices participating was therefore helpful in the achievement of the objectives of the study. Direct observation during practice visits confirmed that the practices were situated in areas with widely differing prevailing socio-economic conditions.

The practitioners also exhibited diverse opinions with regard to the efficacy of prevention, the factors they believed to influence their treatment philosophy and their participation in postgraduate education. Some authorities would disagree with some of the dentists' views, particularly regarding intervals between examinations and the role of dietary counselling. However, the network was formed to develop critical appraisal and research skills rather than

\begin{tabular}{|c|c|c|c|c|c|c|}
\hline \multirow{2}{*}{$\begin{array}{l}\text { Sub-scale } \\
\text { Chewing }\end{array}$} & \multirow{2}{*}{$\begin{array}{c}17-35 \\
0.13(0.46)\end{array}$} & \multirow{2}{*}{$\begin{array}{c}36-50 \\
0.28(0.89)\end{array}$} & \multicolumn{2}{|c|}{$51-60$} & $61-80$ & \multirow{2}{*}{$\begin{array}{c}\text { Kruskall-Wallis test } P \text {-value } \\
0.005\end{array}$} \\
\hline & & & 0.08 & $0.32)$ & $0.35 \quad(0.88)$ & \\
\hline Speaking & $0.14 \quad(0.47)$ & $0.13(0.47)$ & 0.22 & $(0.65)$ & $0.21 \quad(0.66)$ & 0.795 \\
\hline Pain & $1.15 \quad(0.37)$ & $0.99(1.24)$ & 0.85 & (1.17) & $0.72 \quad(1.02)$ & 0.006 \\
\hline Symptoms & 1.59 (1.53) & $1.24(1.47)$ & 1.43 & (1.59) & $1.21 \quad(1.16)$ & 0.091 \\
\hline Eating & $3.97 \quad(1.61)$ & $3.95(1.43)$ & 3.96 & (1.22) & $4.09 \quad(1.71)$ & 0.508 \\
\hline Social & 0.49 (0.73) & $0.49(0.70)$ & 0.55 & $(0.72)$ & $0.43 \quad(0.78)$ & 0.115 \\
\hline Living & $6.53 \quad(1.31)$ & 6.53 (1.59) & 6.31 & $(0.93)$ & $6.32 \quad(0.97)$ & 0.445 \\
\hline Worry & $4.11 \quad(2.07)$ & $3.92(1.84)$ & 3.78 & $(1.67)$ & 3.39 (1.60) & 0.022 \\
\hline Total & $18.34(6.03)$ & 18.01 (6.39) & 17.4 & (5.30) & 16.39 (5.09) & 0.092 \\
\hline
\end{tabular}




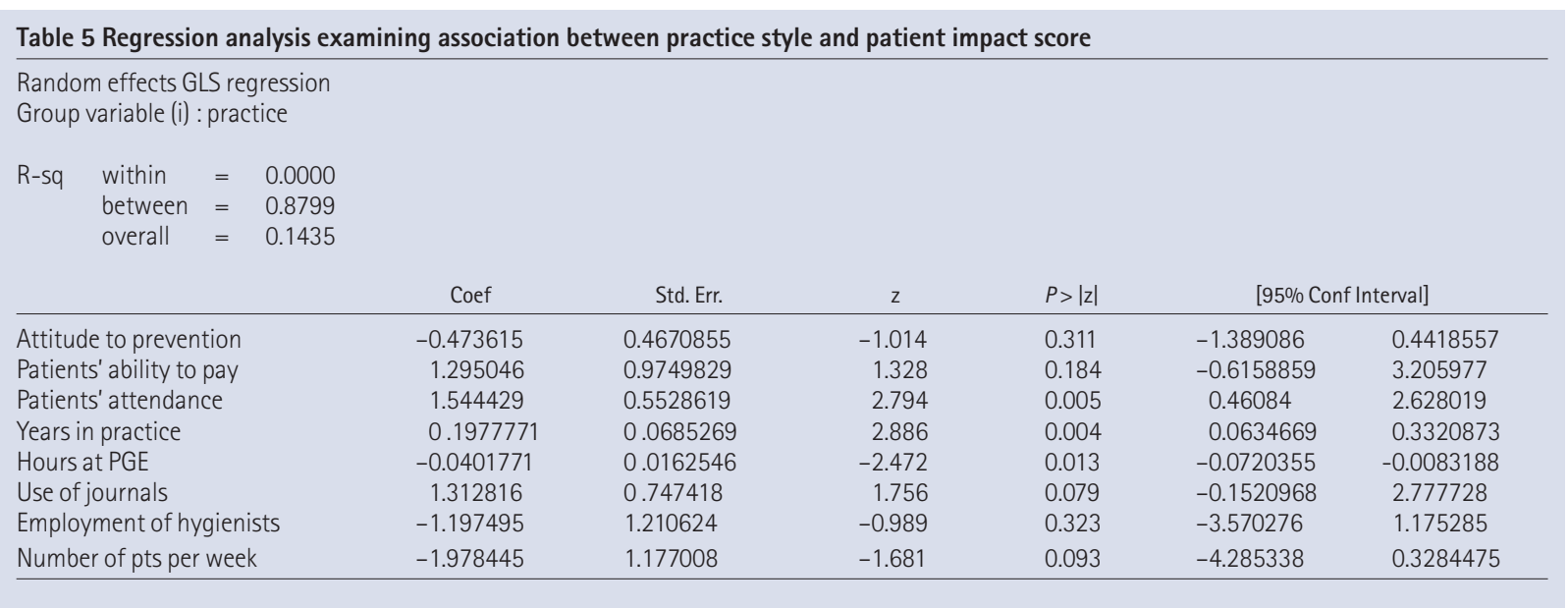

to change practitioners' knowledge and beliefs through didactic teaching.

The response rate of 96\% was extremely satisfactory and the sample of patients, although not representative of the population as a whole, is considered large enough to be reasonably representative of dental practice attenders in the North West area. Comparison of this $96 \%$ response with the rates in studies where patients are approached directly by researchers, rather than by the practitioner who provides their care, indicates that practitioner networks offer a highly efficient and effective route by which the effectiveness of services may be researched and evaluated.

The point prevalences of inability to chew (11\%), sensitivity $(33 \%)$ and pain (23\%) are quite surprising. These figures reveal that a sizeable minority of dentally aware patients have problems which impact on patients' day-to-day life and well-being. For example, around one third had experienced social impacts such as limitation of food choice, dissatisfaction with personal appearance and avoidance of leisure and socialising due to oral problems. This is of concern, as it has been previously shown that oral health is significantly associated with patients' psychological well-being and life satisfaction. ${ }^{4}$ Indeed, the degree of worry and concern about oral health and appearance in this population of dental patients is notable. The high figures for worry (55\% re: appearance, 65\% re health) may reflect a high level of oral health problems. However, an alternative explanation would be that this is a population who are highly dentally motivated and to whom the importance of oral health has been highlighted by their dentist. They may therefore be more concerned about their mouths than the general population. The figures do compare unfavourably with previous surveys of oral health impact carried out on groups who did not necessarily attend dental services. ${ }^{9}$ The measure may therefore have potential as a predictor of service uptake. However, longitudinal follow-up will be important as it has been shown previously that oral health impacts lessen over time in those who receive dental services. ${ }^{10}$

The finding that chewing difficulties are predicated mainly in the older (and denture wearing) patients accords with findings in other studies. ${ }^{11,12}$ Similarly, Macfarlane et al. ${ }^{13}$ have previously reported that, as in this study, older patients report less pain and less concern with regard to oro-facial pain. This finding may be due to an age cohort effect, in that older generations have lower expectations and a generally more stoical and uncomplaining outlook. Alternatively, the ageing of the dentition (reduced pulp chambers, occluded dentinal tubules and less standing teeth) may explain this observation, ie younger people may actually experience more dentally related pain. However, such a clinically oriented explanation could not so easily be applied to the age differences in the degree of worry and concern expressed, which are also higher in the younger age groups.
The research had planned to investigate the relationship between the dentists' treatment attitudes and the patients' subjectively assessed health. That is, the hypothesis was that the dentist's views about the success rates of his treatments, would be likely to affect the type of care the patients received ${ }^{14}$ and, therefore, the impact their oral health had upon them. This hypothesis was supported by the fact that the dentists in this study who believed that the amalgams they placed were short lived were likely to have a patient group with higher oral health impacts. This could mean that dentists who repair and restore frequently have a detrimental effect on patients' subjective oral health status. Similarly, the more importance dentists placed on the patients' dental attendance pattern as a guide to treatment planning, the lower her or his patients' mean impact score. This indicates that dentists who plan treatment, taking their patients' likely behaviours and actions into account, rather than simply making decisions based on clinical findings, have patients who feel happier with their mouths and experience less impact from oral health problems. For example, dentists who take full account of the patients' future attendance when deciding whether to restore a tooth, seem to be acting to the patients' benefit.

Dentists' treatment decisions have previously been shown, in vitro, to be influenced by their feelings about the sensitivity and specificity of the decision making. ${ }^{15}$ This study did not confirm this experimental association, and there was no evidence that attitudes to treatment threshold setting affected patient well-being.

As is common in behavioural and biological research, the explanatory regression model accounted for a small proportion of the variation in observations. The association between years in practice and higher patient impact is probably due, at least in part, to the fact that older dentists will tend to have an older group of patients who, because of the 'cumulative' nature of dental disease, are likely to have more periodontal disease and fewer standing teeth. It would seem unlikely that dentists become less proficient at addressing their patients' subjective needs as they get older (which would also explain the observations but seems less appealing than the age cohort explanation).

Attendance at postgraduate education appeared in the regression analysis to be negatively associated with high subjective patient impacts. It is important to note when interpreting such a finding to remember that the hours of attendance were estimated by the practitioners rather than being obtained from a central source. Such reported data may be unreliable. Furthermore, subjective estimates are known to under or over state the importance of an attribute, particularly if it is an attribute known to be of particular importance to those asking 
the question. Since the research network was partly supported by the postgraduate deanery, it would be very surprising if the number of hours of postgraduate activity had been under estimated.

This paper has described subjective oral health impacts in a population of 718 general practice patients. Fourteen per cent of the differences in mean overall impact in each practice can be explained by practice/practitioner attributes. The number of dentists involved was small and the number of patients per practice limited to fifty. Thus, any significant findings are likely to be real differences, which have clinical significance, as the sample size is not large enough to detect small and possibly irrelevant differences. The sample size may have meant that other factors which have a real but small effect on patients' subjective feelings were not revealed by the research. The study therefore needs to be repeated with larger numbers of dentists to confirm the findings presented here. Such research could then detect factors which affect patient well-being in small and more subtle ways.

The research undertaken by the network has generated a unique dataset from a very large number of general practice patients and has revealed some important and interesting associations between dentists' practice 'styles' and the impact oral health has on patients. The next step in this programme of research, should funding allow, is the longitudinal follow-up of the 718 involved patients. This will allow the oral health gain (or loss) contingent upon dental treatment (or lack of it) to be investigated.
1. Kay E J, Nuttall N. Clinical decision making. An art or a science? London: British Dental Association Books, 1977.

2. Kay E J, Ward N, Locker et al. A general dental practice research network. Philosophy, activities and participant views. Br Dent J 2003: 194: 545-549.

3. Griffiths F, Wild A, Harvey J, Fenton E. The productivity of primary care research networks. BrJ Gen Pract 2000; 50: 913-915.

4. Gift $H$, Atchkinson K. Oral health, health and related quality of life. Med Care 1995: 33: 57-77.

5. Slade G D ed. Measuring oral health and quality of life. Chapel Hill: University of North Carolina, 1997.

6. Slade G, Spencer A. Development and evaluation of the oral health impact profile. Comm Dent HIth 1994: 11:3-11.

7. World Health Organisation. International classification of Impairments, disabilities and handicaps: a manual of classification. Geneva: World Health Organisation, 1980

8 Locker D, Clarke M, Payne. Self perceived oral health status psychological wellbeing, and life satisfaction. J Dent Res 2000; 79: 970-975.

9. Tickle $M$, Craven $R$, Worthington H V. A comparison of the subjective oral health status of older adults from deprived and affluent communities. Comm Dent Oral Epidemiol 1997; 25: 217-22.

10. Locker D. Does dental care improve the oral health of older adults? Comm Dent HIth 2001: 18: 7-15.

11. Leake J L, Hawkins R, Locker D. Social and functional impact of reduced posterior dental units in older adults. J Oral Rehab 1994; 21: 1-10.

12. Smith J M, Sheiham A. How dental conditions handicap the elderly. Comm Dental Oral Epidemiol 1979: 7: 305-310.

13. Macfarlane TV, Gray R J M, Kincey J, Worthington H V. Oral Diseases 2001; 7: 321-330.

14. Kay E, Knill-Jones R. Variation in restorative treatment decisions: application of ROC analysis. Comm Dent Oral Epidemiol 1992; 20: 113-117.

15. Kay E, Nuttall N M. Relationship between dentists' treatment attitudes and their restorative dental treatment decisions planned on the basis of bitewing radiographs. Comm Dent Oral Epidemiol 1993; 21: 43-46. 